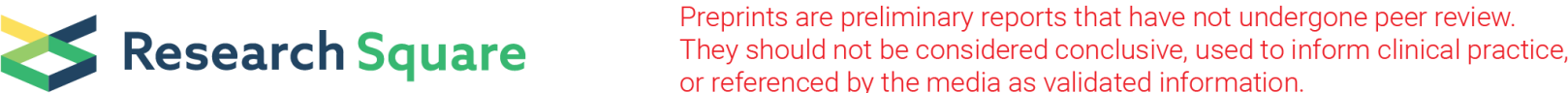

\section{The Effect of Tranexamic Acid and Epsilon- Aminocaproic Acid on Bleeding During Hip and Knee Arthroplasty囚A Retrospective Study}

\section{Xu Xiaoxiao}

Chongqing Health Center for Women and children

Xu Mingdeng (D304669329@qq.com )

The Third Affiliated Hospital of Chongqing Medical University

\section{Xie Feng}

The Third Affiliated Hospital of Chongqing Medical University

\section{Ma Jikun}

The Third Affiliated Hospital of Chongqing Medical University

\section{Wang Xin}

The Third Affiliated Hospital of Chongqing Medical University

\section{Jiang Dianming}

The Third Affiliated Hospital of Chongqing Medical University

\section{Research Article}

Keywords: Tranexamic acid, Epsilon-aminocaproic acid, Arthroplasty, Bleeding, Thrombus

Posted Date: December 6th, 2021

DOI: https://doi.org/10.21203/rs.3.rs-1117640/v1

License: (c) (i) This work is licensed under a Creative Commons Attribution 4.0 International License. Read Full License 


\section{Abstract}

\section{Purpose}

To compare the hemostatic effect and risk of thrombosis between tranexamic acid and epsilonaminocaproic acid.

\section{Methods}

A retrospective study of 140 cases of joint replacement, including 93 cases in the tranexamic acid (TXA) group and 47 cases in the epsilon-aminocaproic acid (EACA) group. TXA or EACA was injected intravenously 30 minutes before surgery, and TXA or EACA was infused into the joint cavity after the wound was closed. The drainage, blood loss and plasma albumin loss were observed after operation.

Results

The postoperative hemoglobin loss in the two groups was $19.1 \pm 11.4 \mathrm{~g} / \mathrm{L}, 20.3 \pm 13.6 \mathrm{~g} / \mathrm{L}, \mathrm{P}>0.05$. However, the drainage volume of the TXA group was less than that of the EACA group, which were $103.3 \pm 92.1 \mathrm{ml}$ and $117.4 \pm 120.9 \mathrm{ml}$, respectively, $P<0.05$. The blood transfusion rate in the TXA group was higher than that in the EACA group, $14 \%$ and $34 \%$, respectively, $P<0.05$. The postoperative plasma albumin loss of the two groups of patients was $7.4 \pm 8.0 \mathrm{~g} / \mathrm{L}$ and $7.3 \pm 5.5 \mathrm{~g} / \mathrm{L}$ respectively, $\mathrm{P}=0.05$.

\section{Conclusions}

The hemostatic effect of TXA was slightly better than that of EACA, and the proportion of transfusion of TXA was lower.

Level of Evidence: Level III

\section{Introduction}

For patients with moderate to severe degenerative knee or hip disease and osteoarthritis pain, knee/hip replacement is a reliable surgical procedure, but perioperative bleeding is the main challenge for surgeons. A number of previous studies have reported that the estimated blood loss was between 800 and $1800 \mathrm{ml}[1,2]$. Large blood loss in patients leads to swelling, hematoma formation, postoperative stiffness, and may prolong drainage time, delay recovery, functional recovery, and discharge, and increase medical costs. These characteristics increase the risk of postoperative periprosthetic joint infection, transfusion-related acute lung injury, transfusion-related circulatory overload, hemolytic transfusion reactions, delayed functional recovery, prolonged hospital stay, and even the risk of death[3].

TXA is a synthetic analogue of amino acid lysine that acts on lysine plasminogen by competitively blocking lysine binding sites, resulting in inhibition of fibrinolysis [4]. Previous meta-analyses have shown that both topical and intravenous TXA were effective in reducing total blood loss in TJA patients. EACA is 
another anti-fibrinolytic drug that is also used in surgery. Breda et al.[5] reported that local administration of caproic acid after cardiac surgery reduces postoperative bleeding and the need for blood transfusion.

The objective of this study was to compare the effects of TXA and EACA in hip and knee replacement.

\section{Materials And Methods}

A retrospective study compared TXA versus EACA wound drainage and blood transfusion rates during hip or knee replacement. All patients were given TXA injection $1 \mathrm{~g}$ or EACA injection 1g intravenously 30 minutes before operation, and after suture the wound, the joint cavity was infused with TXA injection $2 \mathrm{~g}$ or EACA injection $2 \mathrm{~g}$. Hemoglobin and plasma albumin were reexamined on the first day after surgery. The drainage tube was removed 24 hours after surgery and the drainage volume was recorded. Low molecular weight heparin was given to prevent thrombosis 12 hours after operation. Patients who did not use TXA or EACA, who did not place drainage, or who did not review hemoglobin and albumin on the first postoperative day were excluded.

This retrospective study was approved by the Ethics Committee of the Third Affiliated Hospital of Chongqing Medical University.

The data collected include gender, age, drug use (TXA or EACA), drainage volume, preoperative and postoperative hemoglobin and albumin, and blood transfusion volume, and thrombosis cases.

\section{Statistical Analysis}

The SPSS version 17.0 statistical package for Windows 10 was used for the statistical analysis. All results were recorded in a computer database for subsequent cleaning and statistical analysis. An initial description of the main characteristics was performed. For quantitative data, when appropriate, we calculated mean and standard deviation (SD) and for categorical variables as frequencies and percentages. Qualitative data such as gender and blood transfusion rate were subjected to chi-square test. Quantitative data such as age, drainage volume, blood loss, plasma albumin, operation time, and plasma albumin loss were performed using the Wilcoxon test. A p-value $\leq 0.05$ was considered statistically significant.

\section{Results}

Among the patients who underwent hip and knee arthroplasty in our hospital from February 2019 to March 2021, with 47 in the EACA group, 93 in the TXA group, 10 patients without drainage tube were excluded, and 6 patients were excluded without rechecking hemoglobin and plasma albumin on the first postoperative day, 6 patients were excluded without using TXA or EACA.. All operations were performed by two surgeons. 
The average age of patients in the TXA group and the EACA group was 70.0 years and 71.1 years, respectively(Table 1). The Wilcoxon test showed $P>0.05$, and there was no statistical difference in age between the two groups. The female patients in TXA group accounted for $60.2 \%$, and the female patients in caproic acid group accounted for $70.2 \%$. The Chi-square test showed that $P>0.05$, showing no statistical significance. Preoperative hemoglobin in the TXA group was slightly higher than that in the EACA group, but the Wilcoxon test showed $P>0.05$, and there was no statistical difference between the two groups. The preoperative plasma albumin of the TXA group was slightly higher than that of the EACA group, but the Wilcoxon test showed $P>0.05$, there was no statistical difference between the two groups.

The postoperative drainage volume in the EACA group was more than that in the TXA group. The Wilcoxon test showed $\mathrm{P}<0.05$, which was statistically significant(Table 2 ). The blood loss of the EACA group was slightly more than that of the TXA group, but the Wilcoxon test showed P>0.05, which was not statistically significant. The operation time in the TXA group was slightly longer than that in the caproic acid group, but the Wilcoxon test showed that the operation time in the two groups was not statistically significant $(P>0.05)$. There were 13 patients in the TXA group who received intraoperative or postoperative blood transfusion, and 16 patients in the caproic acid group who received blood transfusion, accounting for $14 \%$ and $34 \%$, respectively. Chi-square test of data in the two groups showed $\mathrm{P}<0.05$, which was statistically significant. Thrombus was formed in 8 cases in TXA group and 4 cases in caproic acid group. Chi-square test showed $\mathrm{P}>0.05$ in both groups, showing no statistical significance.

\section{Discussion}

Specifically, TXA prevents the formation of plasmin and its degradation of fibrin by competitively binding to plasminogen lysine receptor sites. In high doses, it can also have a protective effect on platelets. EACA is a 6-aminocaproic acid, which also belongs to the antifibrinolytic drug of the lysine class. Thus, EACA is believed to work by a similar mechanism to TXA.

Excessive intraoperative blood loss can easily lead to hypotension and other heart, lung and kidney complications, and have a chain adverse effect on perioperative rehabilitation. Excessive bleeding in the early postoperative period can lead to hematoma formation, swelling, stiffness and wound complications, and affect the long-term efficacy of joint replacement surgery. In addition, there are reports that blood transfusion after joint replacement can cause acute lung injury, infection, immunosuppression, and allergic reactions[6].

A review concluded that TXA does not increase the risk of death, myocardial infarction, cerebrovascular accident, deep vein thrombosis, PE, or kidney damage6. Hobbs et al. retrospectively evaluated the effect of EACA in primary THA and TKA [7]. They found that the hemoglobin loss was significantly lower in the EACA group $(2.7+0.8 \mathrm{mg} / \mathrm{dl})$ compared with the control group (no antifibrinolytic, $3.4+1.1 \mathrm{mg} / \mathrm{dl})(P$ $<0.05)$. The incidence of blood transfusion in the EACA group (2.7\%) was also significantly lower than that in the control group $(25.4 \%)(P<0.05)$. Our study showed that the drainage volume, hemoglobin loss, and age of patients in the TXA group were not statistically significant compared with theEACA group, but 
the drainage volume and hemoglobin loss in theTXA group were still lower than those in the EACA group. The age of transfusion patients in TXA group was older than in EACA group. The blood transfusion ratio of the TXA group was significantly lower than the EACA group, which was statistically significant. Therefore, we believed that the hemostatic effect of TXA was slightly better than EACA.

Postoperative deep vein thrombosis is a common complication of total knee arthroplasty and total hip arthroplasty. DVT can develop into pulmonary embolism (PE), leading to death[8]. Theoretically, TXA and EACA inhibit fibrinolytic activity and may increase the risk of DVT. A stedt et al[9]. found that intravenous TXA does not inhibit the fibrinolytic activity of normal vein walls. A large number of studies have confirmed the safety of TXA and will not increase the incidence of DVT or PE[10,11]. Our study showed that there was no significant increase in thrombus in the two groups of patients, and no PE occurred. No allergic and other adverse reactions were observed during and after operation.In 2014, Poeran et al. retrospectively analyzed the outcome data of 872,416 TKA or THA patients and found that after the use of TXA, thromboembolic events and kidney damage did not increase[12]. Our study showed that there was no significant increase in thrombus in the two groups of patients, and no pulmonary embolism occurred. No allergic and other adverse reactions were observed during and after operation.

\section{Conclusions}

The hemostatic effect of TXA was slightly better than that of EACA, and the proportion of transfusion of TXA was lower. Rates of deep vein thrombosis were similar in both.

\section{Declarations}

\section{Insufficient}

There were few cases in this study. Hip and knee replacements were not separated. Intraoperative blood loss was not counted. Thrombus examination was performed after the lower limbs were swollen, but routine screening was not performed.

\section{Acknowledgments}

Thank you to my colleagues for their assistance in the operation and thank you to the other authors for their contributions to the research.

\section{Conflict of interest statement}

The authors state that they have no conflicts of interest.

\section{Funding}

No funding. 


\section{Ethical Approval}

The experimental protocol was established, according to the ethical guidelines of the Helsinki Declaration. This study was approved by the Ethics Committee of the Third Affiliated Hospital of Chongqing Medical University.

\section{Consent to Participate}

Written informed consent was obtained from individual or guardian participants.

\section{Consent to Publish}

All data generated or analysed during this study are included in this published article.

\section{Competing Interests}

The authors declare that they have no conflict of interest.

\section{Authors Contributions}

Xu Mingdeng:Conceptualization, Methodology, Software, Supervision.Ma Jikun:Writing- Reviewing and Editing.Xu Xiaoxiao: Data curation, Writing- Original draft preparation.Xie Feng and Wang Xin :Operation.Jiang Dianming: Visualization, Investigation, Validation.

\section{Availability of data and materials}

The datasets used or analysed during the current study are available from the corresponding author on reasonable request.

\section{References}

1. Sehat KR, Evans RL, Newman JH. Hidden blood loss following hip and knee arthroplasty. Correct management of blood loss should take hidden loss into account. J Bone Joint Surg Br86:561565(2004).

2. Rosencher N.et al. Orthopedic Surgery Transfusion Hemoglobin European Overview (OSTHEO) study: blood management in elective knee and hip arthroplasty in Europe. Transfusion 43:459-469 (2003).

3. Hogan CA, Golightly LK, Phong S, Dayton MR, Lyda C, Barber GR. Perioperative blood loss in total hip and knee arthroplasty: Outcomes associated with intravenous tranexamic acid use in an academic medical center. SAGE Open Med 4:2050312116637024(2016)

4. Ambra LF, de Girolamo L, Niu W, Phan A, Spector M, Gomoll AH. No effect of topical application of tranexamic acid on articular cartilage. Knee Surg Sports Traumatol Arthrosc 27:931-935 (2019).

5. Meneghini M. et al. Combining Sensitive Crossmatch Assays With Donor/Recipient Human Leukocyte Antigen Eplet Matching Predicts Living-Donor Kidney Transplant Outcome. Kidney Int Rep 
3:926-938 (2018).

6. Henry DA, et al. Anti-fibrinolytic use for minimising perioperative allogeneic blood transfusion. Cochrane Database Syst Rev :CD001886(2001).

7. Hobbs JC, Welsby IJ, Green CL, Dhakal IB, Wellman SS. Epsilon Aminocaproic Acid to Reduce Blood Loss and Transfusion After Total Hip and Total Knee Arthroplasty. J Arthroplasty 33:55-60 (2018)

8. Foreman PM.et al. Antifibrinolytic therapy in aneurysmal subarachnoid hemorrhage increases the risk for deep venous thrombosis: A case-control study. Clin Neurol Neurosurg 139:66-69(2015)

9. Astedt B, Liedholm P, Wingerup L. The effect of tranexamic acid on the fibrinolytic activity of vein walls. Ann Chir Gynaecol 67:203-205(1978)

10. Gillette BP, DeSimone LJ, Trousdale RT, Pagnano MW, Sierra RJ.Low risk of thromboembolic complications with tranexamic acid after primary total hip and knee arthroplasty. Clin Orthop Relat Res 471:150-154 (2013).

11. Nishihara S, Hamada M. Does tranexamic acid alter the risk of thromboembolism after total hip arthroplasty in the absence of routine chemical thromboprophylaxis. Bone Joint J 97-B:458-462 (2015).

12. Poeran J. et al. Tranexamic acid use and postoperative outcomes in patients undergoing total hip or knee arthroplasty in the United States: retrospective analysis of effectiveness and safety. BMJ 349:g4829(2014)

\section{Tables}

Table 1 Preoperative observation index

\begin{tabular}{|c|c|c|c|}
\hline & TXA & EACA & $\mathrm{P}$ \\
\hline $\mathrm{N}$ & 93 & 47 & \\
\hline Age $(y)$, mean (SD) & $70.0 \otimes 11.9 \rrbracket$ & 71.1ه10.7凶 & $0.182^{\#}$ \\
\hline Female, n (\%) & $56 \rrbracket 60.2 \% \rrbracket$ & $33 \otimes 70.2 \% \rrbracket$ & $0.270^{*}$ \\
\hline Preoperative hemoglobin $₫$ mean(SD) & $123.9 ه 20.5 \rrbracket$ & $118.1 \otimes 19.5 \rrbracket$ & $0.776^{\#}$ \\
\hline Preoperative plasma protein, mean(SD) & $39.0 \rrbracket 4.45 \rrbracket$ & $38.1 \rrbracket 4.25 \rrbracket$ & $0.611^{\#}$ \\
\hline
\end{tabular}

\# Wilcoxon test.

* chi-square test

Table 2 Postoperative observation index 


\begin{tabular}{|c|c|c|c|}
\hline & TXA & EACA & $P$ \\
\hline $\mathrm{N}$ & 93 & 47 & \\
\hline Drainage,mean(SD) & 103.3(92.1) & 117.4(120.9) & $0.001^{\#}$ \\
\hline $\begin{array}{l}\text { Preoperative and postoperative difference of hemoglobin, } \\
\text { mean(SD) }\end{array}$ & 19.1(11.4) & 20.3(13.6) & $0.08^{\#}$ \\
\hline $\begin{array}{l}\text { Preoperative and postoperative difference of plasma } \\
\text { protein, mean(SD) }\end{array}$ & $7.4(8.0)$ & $7.3(5.5)$ & $0.05^{\#}$ \\
\hline Operation time (SD) & $127.0(41.3)$ & $113.9(44.8)$ & $0.870^{\#}$ \\
\hline Patients transfused, $n(\%)$ & $13(14 \%)$ & $16(34 \%)$ & $0.008^{*}$ \\
\hline Transfusion age mean (SD) & $79.1 \otimes 9.7 \otimes$ & $77.8 \otimes 7.8 \rrbracket$ & $0.552^{\#}$ \\
\hline Thrombus $₫ n(\%)$ & $8 \rrbracket 12.9 \% \rrbracket$ & $4 \rrbracket 10.6 \% \rrbracket$ & $0.464^{*}$ \\
\hline
\end{tabular}

\# Wilcoxon test.

* chi-square test 